\title{
Standalone LV Distribution Network Voltage Control Mechanism
}

\author{
Sandro Iacovella, Kris Lemkens, P. Vingerhoets, \\ F. Geth, Geert Deconinck \\ Department of Electrical Engineering \\ K.U.Leuven, ESAT-ELECTA \\ Leuven, Belgium \\ sandro.iacovella@esat.kuleuven.be
}

\author{
Reinhilde D'hulst, Koen Vanthournout \\ Unit Energy Technology \\ VITO, \\ Mol, Belgium \\ reinhilde.dhulst@vito.be
}

\begin{abstract}
This paper describes a voltage stabilizing control mechanism using the available flexibility of smart devices within one household. The flexibility of all types of smart appliances is used, especially smart on/off devices. The main advantage of the developed control system is that it does not require a communication network between the different households, only locally available measurements, such as the household supply voltage, are taken into account. The control system will be rolled out in a real life pilot test. Simulation results point out that the amount of over and under voltage occurrences on average are lowered with $35 \%$.
\end{abstract} grid

Keywords-LV distribution grid, local voltage control, smart

\section{INTRODUCTION}

Over the last decade, there has been a clear focus in the European Union on promoting low-carbon generation technologies and renewables, with new and binding targets of $20 \%$ of power generation from renewable sources by 2020 recently agreed for implementation across Europe. In many countries, feed-in tariffs for eligible technologies have guaranteed returns for investors, and this along with other forms of market support have contributed to a reduction in technology costs and an increasing penetration of smallerscale generation into distribution networks across Europe [1]. The Belgian action plan for renewable energy (NREAP) predicts an increase in the share of renewable energy from 2.6 $\mathrm{GW}$ in 2011 to $11.4 \mathrm{GW}$ in 2020 [2].

Simultaneously, total energy consumption is rising steadily each year by several percent per year [3]. These rates of change will only increase as opportunities for electrification of transport (EV) and heat production (CHP, heat pump, electric hot water buffer) arise as fossil fuels become more expensive and concerns about the emission of greenhouse gases grow [4] [5].

These developments are leading to increasingly complex power flows, pressurizing the European low voltage (LV) distribution networks currently in use. Their historical "fitand-forget" design was consistent with the unidirectional power flows from generator to the end user and their predictable load profiles. Nowadays however, distribution system operators (DSOs) are facing more variable and less predictable power flows, as well as increased (local) peaks in

This work is supported by the Flemish Ministry of Science (Minister I. Lieten) via the project Linear organized by the Institute for Science and Technology (IWT) production and consumption, influencing the (local) voltages which are subject to strict regulation. According to the European EN50160 standard [6], the 10-minute mean rms value of the supply voltage should be $U_{\text {nom }} \pm 10 \%$ for $95 \%$ of the time, measured on a weekly base. Furthermore, the 10minute mean rms value of the Voltage Unbalance Factor (VUF) should be below $2 \%$ for $95 \%$ of the time, measured on a weekly base. Regulating voltage peaks locally could maintain the grid within the acceptable region according to this standard, while minimizing, deferring or even avoiding any capacity upgrades.

Regarding distributed production, many distributed voltage control mechanisms have already been implemented in the inverters of the locally installed distributed energy resources (DERs). The earliest adopted and most rudimental measure consists of country-specific regulations requiring PV inverters to disconnect automatically when a maximum voltage limit is exceeded [7]. This on/off mechanism is currently already required in many countries. Because of the growth in the number of residential PV installations, disconnections already occurring presently in practice will only increase in numbers. This grid security mechanism consequently puts a limit on the amount of PV that can be installed without further grid reinforcements, since this causes a lower yield of the installed $\mathrm{PV}$ installation and thus an increased pay-back period for the owner. In addition, this control mechanism can cause unwanted changes in grid voltage or frequency if there is high PV penetration [8]. A second method to decrease the local voltages peaks is by curtailment of active power injected into the grid according to a piecewise linear droop curve. Instead of fully curtailing the output power when the voltage limit is exceeded, this voltage control mechanism lowers the active output power proportional to the deviation of the grid voltage. A third option to accomplish voltage regulation is the injection of reactive power into the grid [9][10][11]. For a comparison between these three methods see [12]. The increase in energy consumption might also lead to additional under-voltage problems stretching the already problematic feeders to their limits even more. In order to postpone, avoid or delay grid upgrades, several lessons can be learnt from the very related and more mature previously discussed DER integration measures. One solution consists of applying the grid voltage stabilizing load models, such as developed for PV inverters, to 
electric vehicle chargers as well [8]. Similarly, for other inverter-based appliances this method can also be adopted.

The methods discussed above do not require communication between the different smart appliances. Other Demand Side Management strategies are being developed to coordinate different loads and production units to optimize the power flows for a specific objective, e.g. minimal voltage deviations, valley filling or peak shaving [13] [14]. While inherently being able to perform more optimal, these methods still require an (optimal) communication network.

This paper discusses a voltage stabilizing control mechanism for LV distribution networks using the readily available flexibility of the smart devices within one household. The flexibility of all types of smart appliances is used, not only devices having an inverter-like front-end. The main advantage of the control system developed is that it does not require a communication network between the different households within the LV network, nor does it require realtime coordination between households, very fast (and thus expensive) measuring equipment, etc. The developed control system only relies on communication between the different smart appliances within one household, and only uses locally available measurements, such as the household supply voltage. As a result, the proposed control system is easily installed and compatible with DSM infrastructure currently being developed, such as home gateways and smart meters. Moreover, the proposed control system can also be used as a fallback mechanism for other communication-based DSM control systems when communication fails or when the system has been compromised due to cyber security issues [15]. The developed control system will be rolled out in a real life pilot within the LINEAR project [16].

This paper is organized as follows. Section II describes the specifications of the developed control system. Section III explains how the developed control system works. Section IV gives simulation results, and the conclusion of the work are given in Section $\mathrm{V}$.

\section{SYSTEM SPECIFICATIONS}

The developed control system will be implemented and tested in a real life pilot test within the LINEAR project [16] from mid-2013 until mid-2014. Having the control system to be rolled out within existing households, and the requirement to use readily available equipment has a number of consequences in terms of control system specifications and design choices that were made.

All measurements, both local voltage measurements as well as energy measurements, are only available on a quarterhour basis. Also, device control actions are only sent each $15 \mathrm{~min}$. Still, the main goal of the local control system is to mitigate over-and under voltages, defined as voltages with a 10 -min mean rms value falling outside $\pm 10 \%$ of $U_{\text {nom. }}$ As a consequence, the control system will not be able to perform correcting actions within the $10 \mathrm{~min}$ timeframe. However, it can be assumed that a rather large correlation exists between the household load of consecutive time steps [17]. Thus, one may also assume that a relatively large correlation exists between consecutive household supply voltage measurements, justifying basing control actions on previous voltage measurements. All smart appliances that will be managed by the control system are appliances that can only be switched on or off. No power modulating device behavior is possible. The smart appliances that will be used are white good appliances (i.e. dishwashers, washing machines and tumble dryers), electric domestic hot water buffers, and electric vehicles.

A primal requirement of the pilot test is that comfort settings of each household are preserved. This means that, for example, an appliance can never be switched off if the user absolutely requires it to run, even if switching on causes voltage issues.

\section{DISCRETE DROOP CONTROL}

The function of the developed control system, further on called the 'discrete droop control' is to prevent over- and under voltages on a distribution feeder, with only local in-house measurements taken into account. The control system bases his decision to switch on or off a certain smart device on the measured household supply voltage of the previous time step. If an overvoltage is detected, and there are devices available within the household which are able to be switched on, the control system gives the command to switch on. An analogue procedure is followed at undervoltage instants. If no under-or overvoltages are detected, the devices switch on/off at their default behavior. The philosophy behind the developed control system is the well-known droop control, where active and/or reactive power is used to control voltage and/or frequency deviations [18][19]. In the classic droop control within LV networks, only devices having an inverter-like front-end are used, as they are able to react instantaneously, and can change their power output linearly between a minimal and maximal value. Here however, the classic droop control is translated towards on/off switching devices with low reacting times.

\section{A. Device priority}

The priority of a smart appliance is defined as an indication of its state within its flexibility window. When a device urgently needs to switch on to preserve the comfort settings of the user, its priority is high. When it still has some time left before it needs to switch on, its priority is low. Priority is defined as being a number between 0 and 100 .

The device priorities are set to increase linearly to the expiration of the flexibility deadline. The priorities of the smart devices are then calculated according to:

- White good appliances and electric vehicles:

$$
\operatorname{priority}(t)=100 \frac{t-t_{\text {setup }}}{t_{\text {setup }}-t_{\text {deadline }}}
$$

- $\quad$ Electric domestic hot water buffers:

$$
\operatorname{priority}(t)=100 \frac{\operatorname{SoC}(t)-100}{\operatorname{SoC}_{\min }-100}
$$

with $t$, the current time step, $t_{\text {setup }}$, the time at which the user sets up the device, $t_{\text {deadline }}$, the time at which the appliance has to have completed its cycle, SoC, the state of charge of the buffer, and $S o C_{m i n}$, the minimal allowed state of charge of the 
buffer, as set by the user. For an electric vehicle, $t_{\text {deadline }}$ equals the expected time of departure.

\section{B. Switch merit order}

The control system needs to decide which devices to switch on or off. In order to do so, a 'merit order' is defined. The merit order is based on the priorities of the smart appliances, and on the measured voltage. Thus, the developed control system only relies on the available 'state information', no future/past behavior of the appliances is included in the control system calculations, nor does it take into account voltage predictions, etc. This lowers the requirements, and thus the costs, for computational capacity of the in-house control system.

The principle of setting the switch merit order is shown in Fig. 1. It shows that when a certain under or over voltage limit is reached - the Lower and Upper Droop Limits respectively a load will switch on or off. The respective smart appliances are graphically represented by the rectangles L1, L2, L3 and L4. The height of a rectangle represents the power that the load will consume or stop consuming during the next time step. When a voltage higher than the Upper Droop Limit is measured, the device with the highest priority is switch on first. When a voltage is measured below the Lower Droop Limit, the device with the lowest priority is switched off or delayed first. In the example given Fig. 1, L1 has a higher priority than L2. and L3 has a lower priority than L4.

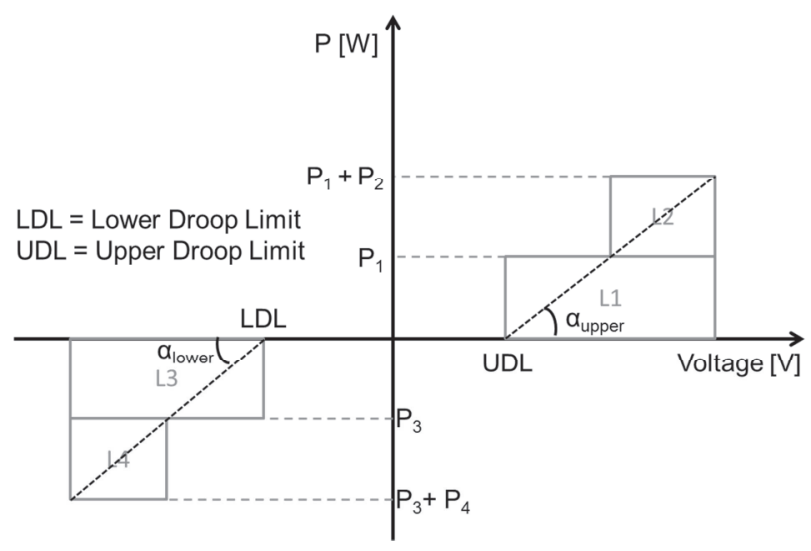

Fig. 1. Schematic overview of switching merit order.

The switching merit order is updated every time step (15 $\min$.).

When the measured voltage falls between the Lower and Upper Droop Limits, the smart appliances fall back to their default behavior. For the white good appliances and the electric vehicles, the chosen default behavior is defined as switching on in the middle of the flexibility window, i.e. halfway between $t_{\text {deadline }}$ and $t_{\text {setup. }}$. For the electric hot water buffers, the default behavior is defined as switching on at a SoC halfway between $\mathrm{SoC}_{\min }$ and a $\mathrm{SoC}$ of $100 \%$, and switching off at a SoC of $100 \%$. In that way maximal flexibility for compensating under-voltages as well as over voltages is available. Note that the switching merit order of devices to switch on at over voltage only contains the devices that have no intention to switch on in their default behavior. Analogously, the switching merit order of devices to switch off at under voltage only contains the devices that have no intention to switch off in their default behavior.

When the priority of a device has reached 100 , i.e. maximal priority, the device always switches on, whatever the measured voltage was. Analogously, when the priority of a device reaches 0 , i.e. minimal priority, the device always switches off. This makes sure that the comfort settings of the user always override control system behavior, a primal requirement within the pilot test.

Design choices to be made when calculating the switching merit order are the definition of the Upper and Lower Droop Limit. Also both the angles $\alpha_{\text {upper }}$ and $\alpha_{\text {lower }}$ (see Fig. 1) need to be chosen in order to define at which measured voltage each smart appliance needs to switch on or off.

\section{SIMULATIONS}

\section{A. Simulation Scenario}

\section{1) Feeder selection}

Simulations were carried out to test the developed control system. The simulations were run on an existing distribution feeder that will be equipped with the control system during the pilot test. The feeder $(230 / 400 \mathrm{~V}$ nominal voltage, $50 \mathrm{~Hz})$ is located in Flanders, Belgium, and has a TT grounding arrangement. The feeder has a three-phase topology, with both underground and overhead connections. The topology is depicted in Fig. 2. All inter house feeder connections up to node 17 are of cable type EAXVB $1 \mathrm{kV} 4 \times 150 \mathrm{~mm}^{2}$, and run underground.

From node 22 to the feeder end, the cable type is EXVB 1 $\mathrm{kV} 4 \times 70 \mathrm{~mm}^{2}$. The cable parameters, provided by the DSO, are listed in TABLE I. . The voltages on the feeder are calculated using a backward-forward sweep static load flow. It holds into account the three-phase unbalance and is implemented in MATLAB [20].

TABLE I. CABLE PARAMETERS

\begin{tabular}{|c|c|}
\hline Cable Type & Impedance at $20^{\circ} \mathrm{C}$ \\
\hline EAXVB $1 \mathrm{kV} 4 \times 150 \mathrm{~mm}^{2}$ & $0.294+0.077 \mathrm{j} \Omega / \mathrm{km}$ \\
\hline EXVB $1 \mathrm{kV} 4 \times 70 \mathrm{~mm}^{2}$ & $0.268+0.249 \mathrm{j} \Omega / \mathrm{km}$ \\
\hline BUAL $1 \mathrm{kV} \mathrm{4 \times 70} \mathrm{mm²}$ & $0.443+0.353 \mathrm{j} \Omega / \mathrm{km}$ \\
\hline
\end{tabular}

\section{2) Smart households}

Fig. 2 shows that 15 out of the 38 households are smart households, i.e. one or more smart appliances are installed in the households. TABLE II. indicates to which grid nodes the smart households are located, and gives the lengths of the cables used to connect the households to the feeder. Columns WM, DR, DW and HWB indicate the presence of a controllable washing machine, tumble dryer, dishwasher and/or electric hot water buffer respectively. A total of 4 electric vehicles are also present as smart appliances, their location however is not fixed: during the pilot test, the vehicles switch 


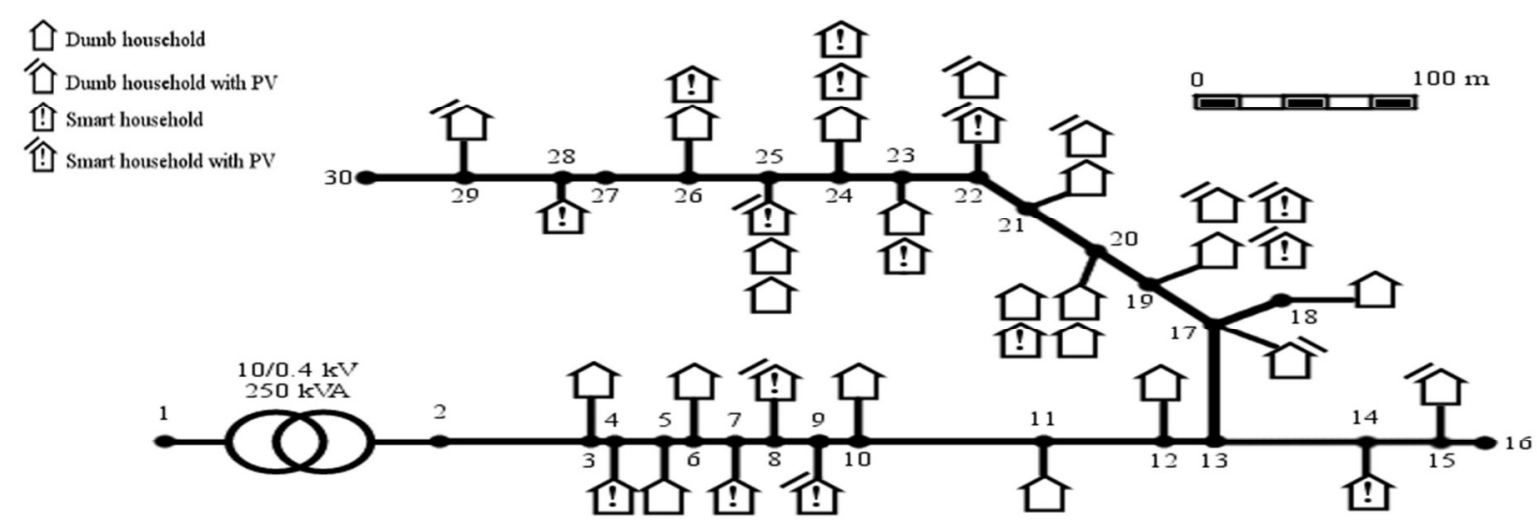

Fig. 2. Feeder topology selected for simulations, the inter house connections are drawn to scale.

owners each few weeks. Twelve households have a PV installation, their size is also given in TABLE II. .

\section{3) Profiles}

\section{a) PV installations}

PV Installations larger than $5.9 \mathrm{kWp}$ are assumed to be three-phase connected, as required by regulation. The PV profiles used are derived from measurements on one existing installation. Then, the PV profile is scaled to match the $\mathrm{kWp}$ sizes shown in TABLE II.

\section{b) Household Load Profiles}

Each simulation, a random set of 38 load profiles are selected from an available set of 100 measured household load profiles. The available load profiles are statistically representative for the population in Belgium, and were measured on a $15 \mathrm{~min}$. basis. Only the active power was measured, so reactive power is neglected in the simulations. All smart appliance profiles (white good, EV, electric hot water buffer), as well as PV profiles are added up to these household profiles where applicable.

TABLE II. HOUSEHOLDS EQUIPPED WITH SMART APPLIANCE(S) AND/OR PV INSTALLATION

\begin{tabular}{|l|l|l|l|l|l|l|l|}
\hline House & Node & Dist [m] & WM & DR & DW & HWB & PV [kWp] \\
\hline \hline 2 & 4 & 17 & $\mathrm{x}$ & & $\mathrm{x}$ & & \\
\hline 5 & 7 & 15 & $\mathrm{x}$ & $\mathrm{x}$ & $\mathrm{x}$ & & \\
\hline 6 & 8 & 15 & $\mathrm{x}$ & $\mathrm{x}$ & $\mathrm{x}$ & & 3,5 \\
\hline 7 & 9 & 13 & $\mathrm{x}$ & $\mathrm{x}$ & $\mathrm{x}$ & $\mathrm{x}$ & 5,6 \\
\hline 11 & 14 & 13 & & & $\mathrm{x}$ & & \\
\hline 12 & 15 & 13 & & & & & 5 \\
\hline 14 & 17 & 14 & & & & & 11,3 \\
\hline 16 & 19 & 35 & $\mathrm{x}$ & & $\mathrm{x}$ & $\mathrm{x}$ & $4,2+14,3$ \\
\hline 17 & 19 & 35 & & & & & 7,5 \\
\hline 18 & 19 & 25 & $\mathrm{x}$ & $\mathrm{x}$ & $\mathrm{x}$ & & 4,6 \\
\hline 20 & 20 & 15 & $\mathrm{x}$ & & & & \\
\hline 23 & 21 & 25 & & & & & 2,8 \\
\hline 25 & 22 & 25 & & & & & 5,6 \\
\hline 26 & 22 & 20 & & $\mathrm{x}$ & & $\mathrm{x}$ & 5,8 \\
\hline 27 & 23 & 35 & $\mathrm{x}$ & $\mathrm{x}$ & $\mathrm{x}$ & $\mathrm{x}$ & \\
\hline 30 & 24 & 25 & $\mathrm{x}$ & $\mathrm{x}$ & & & \\
\hline 31 & 24 & 30 & $\mathrm{x}$ & $\mathrm{x}$ & & $\mathrm{x}$ & \\
\hline 32 & 25 & 27 & $\mathrm{x}$ & & $\mathrm{x}$ & & 5,9 \\
\hline
\end{tabular}

\begin{tabular}{|l|l|l|l|l|l|l|l|}
\hline 36 & 26 & 15 & $x$ & $x$ & & & \\
\hline 37 & 28 & 20 & $x$ & $x$ & & $x$ & \\
\hline 38 & 29 & 20 & & & & & 5,4 \\
\hline
\end{tabular}

\section{c) Smart appliances: white goods}

The power profiles of the dishwasher, washing machine and tumble dryer are based on synthetic models of the respective appliances [21]. These white good appliances are smart appliances in the sense that their start time can be postponed. When the user sets up the devices, he/she needs to provide a deadline, i.e. when the device needs to have completed its cycle. In the simulations is assumed that the users postpone the deadline randomly, with a normal variation with a mean of 4 hours and a standard deviation of 1 hour. The setup times of the white good appliances is assumed to vary according to measured setup times of the respective white good appliances in a submetering campaign. In the pilot test, the smart white good appliances are unable to interrupt their running cycle.

\section{d) Electric Vehicles}

As previously mentioned, a total of 4 electric vehicles are randomly distributed over the smart households. The vehicles are assumed to have a single-phase connection. Their arrival and departure times are based on an availability study, based on Flemish mobility behavior, given in [22]. It is assumed that the EVs only charge at home and are plugged in each time they arrive there. The discrete charging power and battery capacity are identical to the specifications of the Renault Fluence [23], which will be used in the pilot test. In the pilot test, the charging of the electric vehicle can be paused at any instant.

\section{e) Electric domestic hot water buffer}

The electric hot water buffer is modeled after [24]. The buffer parameters used for modeling are those of a Siemens DF2017 as this is the buffer deployed in the pilot test. The buffers in the pilot test are able to switch on or off at any instant, provided that the State of Charge (SoC) does not fall below the minimal allowed SoC, or rises above a SoC of $100 \%$. In [24] is explained how the $\mathrm{SoC}$ of the electric hot water buffer is calculated. 


\section{B. Simulation Results}

The effectiveness of the developed discrete droop control method is measured by counting the number of over and undervoltage occurrences at the smart households during a number of simulation runs. An overvoltage occurrence is defined as a measured supply voltage higher than $U_{\text {nom }}+10 \%$, an undervoltage occurrence is defined as a measured supply voltage lower than $\mathrm{U}_{\text {nom }}-10 \%$.

The design parameters of the discrete droop control as tested in the simulation are given in TABLE III. The Lower and Upper Droop Limits were chosen to be asymmetric around

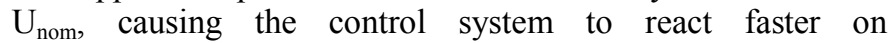
undervoltages than on overvoltages. As overvoltages are mainly caused by power production of the PV installations, the largest overvoltages are found around midday. To prevent the control system to react too soon, i.e. when the maximal overvoltage is not yet reached, the UDL is set to a higher value.

TABLE III. DISCRETE DROOP CONTROL PARAMETERS

\begin{tabular}{|c|c|}
\hline Parameter & Value \\
\hline Lower Droop Limit (LDL) & $4 \%$ of $\mathrm{U}_{\text {nom }}$ \\
\hline Upper Droop Limit(UDL) & $8 \%$ of $\mathrm{U}_{\text {nom }}$ \\
\hline$\alpha_{\text {lower }}$ & $\tan ^{-1}\left(\frac{\sum P_{\text {devices }}}{0.1 U_{\text {nom }}-L D L}\right)$ \\
\hline$\alpha_{\text {upper }}$ & $\tan ^{-1}\left(\frac{\sum P_{\text {devices }}}{0.1 U_{\text {nom }}-U D L}\right)$ \\
\hline
\end{tabular}

A total number of 25 simulation runs were carried out both for a winter day and a summer day (i.e. a day with a lot of PV production), both with and without the discrete droop control in effect. Over all simulation runs, the behavior of the smart white good appliances, the location and charging times of the electric vehicles and the non-flexible load profiles are randomized. The number of over and undervoltage occurrences is also counted in an optimal benchmark situation. To calculate the benchmark, an optimization was performed with the objective to minimize the variance of the voltages along the feeder, constraint to the overall flexibility of all smart appliances. This minimization problem is tackled as a sequential quadratic optimization [25], where the optimal running cycles of each smart appliance are calculated sequentially, this process is iterated until convergence is reached. The benchmark is a theoretical optimum, that can never be reached without having communication and coordination available between the different smart households. It however gives an impression of the performance of the developed discrete droop control system.

Fig. 3 shows the number of over or undervoltage occurrences for each simulation run, results are shown for the benchmark, the default situation and when the discrete droop control method is used. Fig. 4 shows the average number of over or undervoltage occurrences over all simulation runs. The results show that on average, the discrete droop control method is able to lower the number of over and under voltage events on a summer day with $35 \%$ with respect to the default situation (a mitigation of all over and undervoltages is set equal to reaching the optimal benchmark). On average a lowering of $52 \%$ of the number of undervoltages during a winter day is reached with the discrete droop control (during the winter day, no over voltage occurrences were seen). However, when comparing the particular simulation runs, shown in Fig. 3, it can be seen that the discrete droop control method does not always lower the amount of over and undervoltages. This is due to the fact that coordination between the households is inherently not present, nor is the control method able to take future, and possible worse, situations into account.
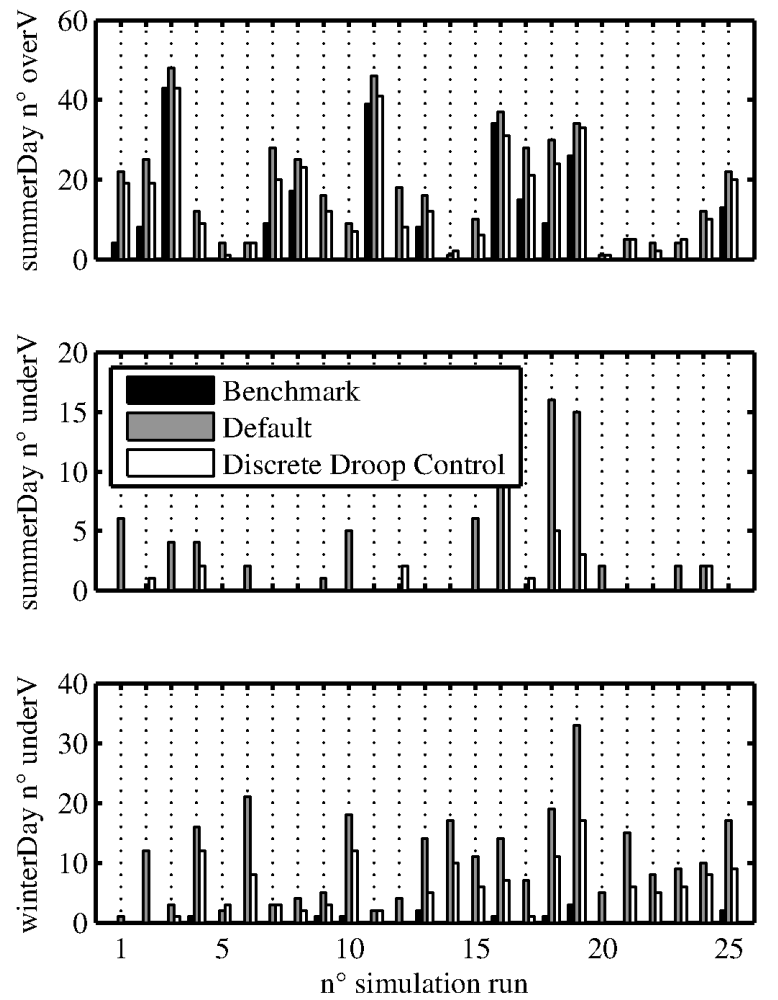

Fig. 3. Total number of overvoltage or undervoltage occurrences per simulation run for a summer day and a winter day.

\section{CONCLUSIONS}

A voltage stabilizing control mechanism using the available flexibility of smart devices within one household is developed. The main advantage of the developed control system is that it does not require a communication network between the different households, nor does it require real-time coordination between households, or very fast (and thus expensive) measuring equipment. Only locally available measurements, such as the household supply voltage, are taken into account. The requirements, and thus the costs, for computational capacity the developed control system are limited. The system will be rolled out in a real life pilot test, requiring the control system to work with smart on/off devices only. The smart appliances that will be used in the pilot are white good appliances (i.e. dishwashers, washing machines and tumble dryers), electric domestic hot water buffers, and electric vehicles. The effect of the developed control system was tested with simulations on an existing LV distribution feeder, taking into account actual smart appliance presence. Simulation 
results point out that the amount of over and under voltage occurrences on average are lowered with $35 \%-52 \%$.

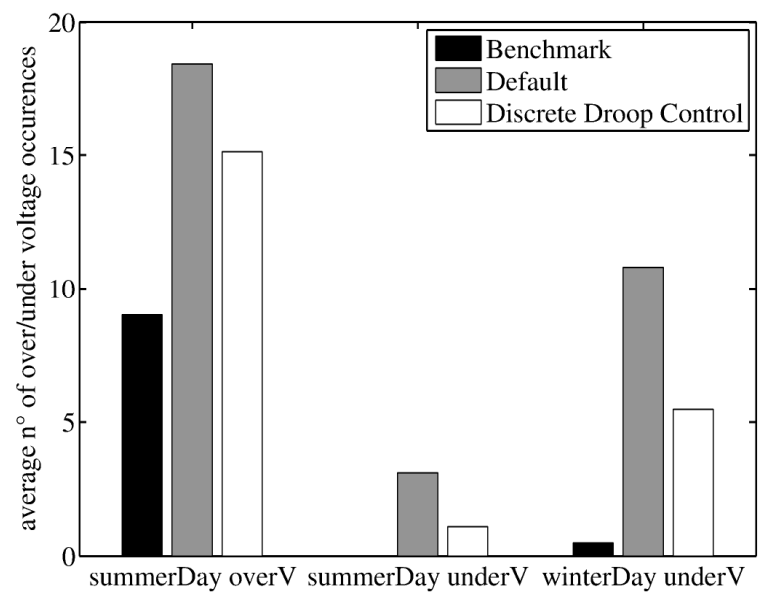

Fig. 4. Average number of over and under voltage occurrences during a summer or winter day for the benchmark, the default situation and when the discrete droop control is used.

\section{REFERENCES}

[1] P. Djapic, C. Ramsay, D. Pudjianto, G. Strbac, J. Mutale, N. Jenkins and R. Allan, "Taking an active approach," Power and Energy Magazine, IEEE, vol. 5, no. 4, pp. 68 - 77, 2007.

[2] "Federal- Regional Energy Consultation Group CONCEREENOVER, "Belgium National Renewable Energy Action Plan"," 2010.

[3] E. E. Agency, "EN18 Electricity Consumption".

[4] J. Kiviluoma and G. Collantes, "Electrification of Energy," Cambridge, Massachusetts, April 23, 2008.

[5] R. Galvin and Y. K., "Perfect Power: How the MicroGrid Revolution Will Unleash Cleaner, Greener,More Abundant Energy," McGraw-Hill Professional, 2008.

[6] "Voltage Characteristics of Electricity Supplied by Public Electricity Networks," CENELEC Std. EN 50 160, Jul. 2010.

[7] M. Braun, T. Stetz, R. Bründlinger, C. Mayr, K. Ogimoto, H. Hatta, H. Kobayash, B. Kroposki, B. Mather, M. Coddington, K. Lynn, G. Graditi, A. Woyte and a. I. MacGill, "Is the distribution grid ready to accept large-scale photovoltaic deployment? State of the art, progress, and future prospects," in Progress in Photovoltaics: Research and Applications, 26th EU PVSEC, Hamburg, Germany, 2011

[8] F. Geth, N. Leemput, J. Van Roy, J. Buscher, R. Ponnette, J. Driesen, "Voltage droop charging of electric vehicles in a residential distribution feeder" in Innovative Smart Grid Technologies (ISGT Europe), 2012 3rd IEEE PES International Conference and Exhibition on, 2012, pp.1-8

[9] K. Büdenbender, M. Braun, M. Stetz and P. Strauss, "Multifunctional PV systems offering additional functionalities and improving grid integration," in Int. J Distributed Energy Resources, vol. 7, no. 2, pp. 109-128, 2011.

[10] B. Bletterie, A. Stojanovic, S. Kadam, G. Lauss, M. Heidl, C. Winter, D. Hanek, A. Pamer, A. Abart: "Local Voltage Control By Pv Inverters: First Operating Experience From Simulation, Laboratory Tests And Field Tests"; in: "Proceedings 27th EUPVSEC", WIP München, Deutschland, 2012.

[11] K. Turitsyn, P. Sulc, S. Backhaus and M. Chertkov, "Options for Control of Reactive Power by Distributed Photovoltaic Generators," Proceedings of the IEEE, vol. 99, no. 6, June 2011.
[12] R. D'hulst and E. Peeters, "Distributed Voltage control Strategies in a LV Distribution Network," in International conference on Renewable Energies and Power Quality, Granada (Spain), 2010.

[13] N. Leemput, J. Van Roy, F. Geth, P. Tant, B. Claessens and J. Driesen, "Comparative Analysis of Coordination Strategies for Electric Vehicles," in Innovative Smart Grid Technologies (ISGT Europe), 2011

[14] P. Samadi, A. Mohsenian-Rad, R. Schober, V. Wong, J Jatskevich, "Optimal Real-Time Pricing Algorithm Based on Utility Maximization for Smart Grid" Smart Grid Communications (SmartGridComm), 2010 First IEEE International Conference on, 2010, pp. $415-420$

[15] A. R. Metke and R. L. Ekl, "Security Technology for Smart Grid Networks," IEEE Transactions on Smart Grid, vol. 1, no. 1, p. 99107, Jun. 2010

[16] B. Dupont, P. Vingerhoets, P. Tant, K. Vanthournout, W. Cardinaels, T. De Rybel, E. Peeters,. R. Belmans, "LINEAR breakthrough project: Large-scale implementation of smart grid technologies in distribution grids" in Innovative Smart Grid Technologies (ISGT Europe), 2012 3rd IEEE PES International Conference and Exhibition on, 2012, pp. 1-8

[17] W. Labeeuw, G. Deconinck, "Customer sampling in a smart grid pilot" Power and Energy Society General Meeting, 2012 IEEE, 2012, pp. 1-7.

[18] K. De Brabandere, B.Bolsens, J. Van den Keybus, J. Driesen, R. Belmans, "A Voltage and Frequency Droop Control Method for Parallel Inverters" in IEEE Transactions on Power Electronics, 2007, vol. 22, pp.1107-1115

[19] J. Vasquez, R. Mastromauro, J Guerrero, M. Liserre, "Voltage Support Provided by a Droop-Controlled Multifunctional Inverter"in Industrial Electronics, IEEE Transactions on, 2009, vol. $56, \mathrm{pp} .4510-4519$

[20] C. Cheng and D. Shirmohammadi, "A three-phase power flow method for real-time distribution system analysis," IEEE Trans. Power Syst., pp. 671-679, 1995.

[21] W. Labeeuw, G. Deconinck, "Non-intrusive detection of high power appliances in metered data and privacy issues", in: EEDAL 2011 - The $6^{\text {th }}$ International Conference on Energy Efficiency in Domestic Appliances and Lighting.

[22] J. Van Roy, N. Leemput, S. De Breucker, F. Geth, P. Tant and J. Driesen, "An Availablilty Analysis and Energy Consumption Model for a Flemish Fleet of Electric Vehicles," European Electric Vehicle Congress (EEVC), pp. 1-12, 2011.

[23] Renault Fluence Z.E. [Online] http://www.renault-ze.com

[24] K. Vanthournout, R. D'hulst, D. Geysen and G. Jacobs, "A Smart Domestic Hot Water Buffer," IEEE Transactions on Smart Grid, pp. 2121-2127, December 2012.

[25] K. Clement-Nyns, E. Haesen, J. Driesen, "The Impact of Charging Plug-In Hybrid Electric Vehicles on a Residential Distribution Grid" IEEE Transactions on Power Systems, 2010, vol. 25, pp $371-380$ 